\title{
Evacuação aeromédica durante a crise sanitária causada pelo SARS-CoV-2 no estado
}

\section{do Amazonas - Brasil}

Aeromedical evacuation during the health crisis caused by SARS-CoV-2 in the state of Amazonas Brazil

Evacuación aeromédica durante la crisis de salud causada por SARS-CoV-2 en el estado de

Amazonas - Brasil

Letícia Lima Borges

ORCID: https://orcid.org/0000-0002-2366-8092 Universidade Federal do Estado do Rio de Janeiro, Brasil E-mail: lima.leticia.borges@gmail.com

Débora Fernanda Haberland

ORCID: https://orcid.org/0000-0001-5448-6278 Universidade Federal do Rio de Janeiro, Brasil E-mail: deborahaber@hotmail.com

Clarissa Coelho Vieira Guimarães

ORCID: https://orcid.org/0000-0002-7713-7182 Universidade Federal do Estado do Rio de Janeiro, Brasil E-mail: guimaraesccv@gmail.com

Luiz Alberto de Freitas Felipe

ORCID: https://orcid.org/0000-0001-8556-7636 Universidade Federal do Estado do Rio de Janeiro, Brasil

E-mail: enfermeiroluizalbertodefreitas@gmail.com

Rafael Abrantes de Lima

ORCID: https://orcid.org/0000-0002-3843-7297 Universidade Federal do Estado do Rio de Janeiro, Brasil E-mail: rafael.abrantes83@gmail.com

Beatriz Gerbassi Costa Aguiar

ORCID: https://orcid.org/0000-0001-6815-4354 Universidade Federal do Estado do Rio de Janeiro, Brasil E-mail: beatriz.costa@unirio.br

\begin{abstract}
Resumo
Objetivo: Descrever experiência da enfermagem militar no preparo de embarque de pacientes em estado crítico do Amazonas para outros estados do Brasil durante a crise sanitária instaurada pela pandemia de COVID-19. Método: Relato de caso construído com a observação da enfermeira de voo sobre a importância do preparo de embarque para a segurança do transporte aeromédico. Discussão: Ainda em solo, a equipe preocupa-se com a configuração da aeronave, com a configuração dos leitos (que acontecerá de forma personalizada), além da sistemática testagem dos equipamentos aeromédicos e reposição de insumo e medicamentos até a aterrisagem em segurança. Conclusão: $O$ planejamento de embarque é uma etapa imprescindível para o transporte seguro de pacientes críticos. O estudo traz uma abordagem dos cuidados específicos no pré-voo da evacuação aeromédica. Neste sentido, o enfermeiro desenvolve as ações de planejamento de embarque, com base na experiência da assistência a pacientes críticos e, participa da equipe multiprofissional durante o voo.

Palavras-chave: Resgate aéreo; Infecção por Coronavírus; Enfermagem militar; Doenças transmissíveis; Enfermagem.

Abstract

Objective: To describe the experience of military nursing in preparing the embarkation of critically ill patients from Amazonas to other states in Brazil during the health crisis caused by the pandemic of COVID-19. Method: Case report built with the observation of the flight nurse about the importance of boarding preparation for the safety of aeromedical transport. Discussion: Still on the ground, the team is concerned with the configuration of the aircraft, with the configuration of beds (which will happen in a personalized way), in addition to the systematic testing of aeromedical equipment and replacement of supplies and medicines until landing safely. Conclusion: Embarkation
\end{abstract}


planning is an indispensable step for the safe transportation of critically ill patients. The study brings an approach to the specific care in the pre-flight of aeromedical evacuation. In this sense, the nurse develops the actions of boarding planning, based on the experience of assistance to critical patients and, participates in the multidisciplinary team during the flight.

Keywords: Air ambulances; Coronavirus infections; Military nursing; Communicable diseases; Nursing.

\section{Resumen}

Objetivo: Describir la experiencia de la enfermería militar en la preparación de embarque de pacientes en estado crítico del Amazonas para otros estados de Brasil durante la crisis sanitaria instaurada por la pandemia de COVID-19. Método: Informe de caso construido con la observación de la enfermera de vuelo sobre la importancia de la preparación del embarque para la seguridad del transporte aeromédico. Discusión: Todavía en tierra, el equipo se ocupa de la configuración de la aeronave, de la configuración de las camas (que se hará de forma personalizada), además de la comprobación sistemática de los equipos aeromédicos y de la reposición de suministros y medicamentos hasta el aterrizaje seguro. Conclusión: La planificación del embarque es un paso esencial para el transporte seguro de pacientes críticos. El estudio aporta un enfoque de los cuidados específicos en el prevuelo de la evacuación aeromédica. En este sentido, la enfermera desarrolla las acciones de planificación del embarque, basándose en la experiencia de asistencia a pacientes críticos y, participa en el equipo multiprofesional durante el vuelo.

Palabras clave: Ambulancias aéreas; Infecciones por Coronavirus; Enfermería militar; Enfermedades transmisibles; Enfermería.

\section{Introdução}

No Brasil, foi declarado, o estado de transmissão comunitária do Coronavírus (COVID-19) em todo o território nacional, no dia 3 de fevereiro de 2020, por meio da Portaria n 188/GM/MS, de 3 de fevereiro de 2020, que declara Emergência em Saúde Pública de importância Nacional (ESPIN) em decorrência da Infecção Humana pelo novo Coronavírus (2019-nCoV) (Lana et al., 2020; Ministério da Saúde, 2020).

O estado brasileiro do Amazonas e sua capital Manaus, tornou-se o epicentro da epidemia com um aumento repentino de mortes que preocupa gestores e sociedade (Ventura et al., 2021; Orellana et al., 2020). Com as elevadas taxas de incidência e mortalidade pela COVID-19 em maio de 2020, causou colapso do sistema funerário durante a primeira onda da pandemia, trazendo sofrimento à sua população. Em dezembro de 2020 e primeiras semanas de janeiro de 2021, nova onda de casos, ocasionou o colapso do sistema municipal de saúde por falta de leitos de enfermaria, leitos de UTI e oxigênio (Barreto et al., 2021; Kerr et al. 2021).

Em seu perfil na rede social, a Força Aérea Brasileira (FAB) publicou que no início de janeiro de 2021, que já havia somado mais de 1.443 horas de voo em apoio à Operação COVID-19, transportadas 1.594 toneladas de carga e 593 pacientes foram transferidos da região Norte para outros estados (Faria, 2021, Rocha 2021).

Por meio do Comando de Operações Aeroespaciais (COMAE), a FAB cumpriu as missões que tiveram como objetivo minimizar os impactos do novo Coronavírus no sistema de saúde de alguns estados do Norte do País. O Transporte Aéreo Logístico da FAB integrou as ações da Operação COVID-19, acionada pelo Ministério da Defesa, em uma cooperação com o Ministério da Saúde. (Força Aérea Brasileira, 2021)

Para remover um paciente crítico por meio aéreo é importante que a equipe de saúde possua experiência profissional uma vez que a decisão pelo transporte será baseada em uma avaliação criteriosa em relação aos benefícios e aos potenciais riscos. Sendo assim, o transporte aéreo tanto por asas rotativas ou fixas é comumente realizado por militares e outros profissionais capacitados (Borges et al., 2022; Beard et al., 2016; Scuissiato et al., 2012; et al., 2011).

Ao planejar o transporte de um paciente crítico, deve-se procurar prever e, se possível, antecipar, todas as intercorrências que possam ocorrer durante o deslocamento, sejam de origem médica, logística, trânsito, condições climáticas ou até da operacionalidade do hospital (Borges et al., 2022; Libardi, 2019).

Além disso, a decisão operacional de transportar pacientes com risco biológico é complexa, pois é influenciada por 
múltiplos fatores, incluindo o agente etiológico envolvido. Ao contrário das vítimas nucleares e químicas, os pacientes com risco biológico podem transmitir a doença mesmo após a descontaminação externa e, como é o caso, é muito mais contagiosa nos estágios finais da doença. (Pérez et al., 2017).

Este estudo justifica-se, pelo destaque e importância do transporte aeromédico no Brasil, no cenário da pandemia de COVID-19, onde militares da saúde da Força Aérea Brasileira, tiveram protagonismo no auxílio e apoio ao estado do Amazonnas, removendo pacientes críticos, fornecendo transporte de suprimentos com o intuito de minimizar o sofrimento da população daquele estado.

E como relevância, a importância de apresentar artigos científicos à comunidade acerca da especialização da enfermagem aeroespacial que é muito recente no País, com a formação da Associação Brasileira de Enfermagem Aeroespacial teve seu surgimento no ano de 2010 na missão de realizar voo mais seguro, eficaz, padronizado e embasado em estudos científicos claros e atuais, com sua normatização reconhecida pelo Conselho Federal de Enfermagem através da Resolução COFEN N 551 , de 26 de maio de 2017, pela Resolução COFEN Nº 656, de 17 de dezembro de 2020 e alterada pela Resolução COFEN N ${ }^{\circ} 660$, de 03 de março de 2021.

Portanto, foi delimitado como objetivo do estudo: descrever experiência da enfermagem militar no preparo de embarque de pacientes em estado crítico do Amazonas para outros estados do Brasil durante a crise sanitária instaurada pela pandemia de COVID-19.

\section{Metodologia}

Trata-se de um relato de caso sobre o transporte aeromédico de pacientes em estado crítico acometidos pelo SARSCoV-2 durante a crise sanitária causada pela sobrecarga do Sistema de Saúde do estado do Amazonas, Brasil. Foram utilizados os registros de bordo realizado pela equipe de Enfermagem, que participou do preparo das missões de transporte aeromédico durante a primeira quinzena de fevereiro de 2021 na Base Aérea de Manaus. Foi realizada uma leitura preliminar do diário escrito pela Enfermeira Militar que participou da operação.

Deste modo, optou-se por descrever as experiências vivenciadas visto que, o relato de caso é um tipo de artigo científico dos mais comuns de apresentação em congressos ou até mesmo em publicações de periódicos no Brasil e no exterior, sendo uma importante fonte informação, fornecendo subsídios para o melhor manejo de pacientes em determinadas situações (Yoshida, 2007).

\section{Relato de Caso}

Com o aumento do número de casos de COVID-19 na capital do estado do Amazonas, foi necessária a implantação da Célula de EVAM-COVID para o apoio aos civis e militares da região. No primeiro momento, militares do Esquadrão Pelicano (sediado em Campo Grande - MS) foi deslocado para Manaus com a finalidade de compor a Célula de EVAM-COVID trazendo a Aeronave C-105 FAB 2810, todo material aeromédico necessário para montar 04 leitos de UTI AÉREA COVID, sendo uma experiência ímpar no transporte aeromédico de pacientes em estado crítico.

A aeronave Airbus Casa C-105 AMAZONAS da Força Aérea Brasileira é um veículo aéreo com capacidade para transportar com segurança 09 pacientes sentados na estação 1 - destacada em amarelo (5 pacientes nos assentos do lado direito da aeronave e 4 pacientes do lado esquerdo). Para o transporte de pacientes em maca, essa aeronave terá capacidade de 15 lugares disponibilizados em três níveis de altura nas estações 2, 3 e 4 - destacadas em vermelho (Força Aérea Brasileira, 2011). 
Figura 1. Croqui da aeronave Airbus Casa C-105 - Amazonas. Brasília, DF, 2011.

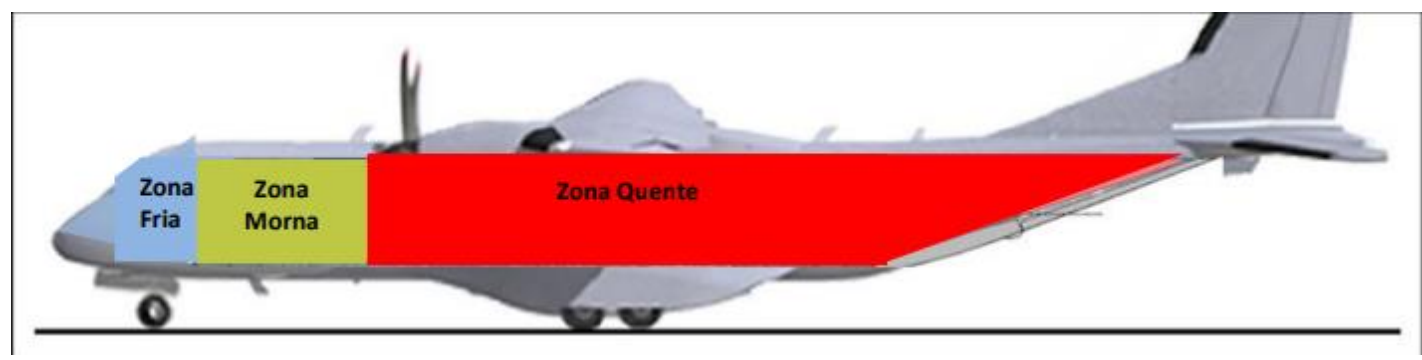

Fonte: Força Aérea Brasileira (2011).

No dia 08 de fevereiro de 2021, iniciou-se a experiência da enfermeira de bordo na Célula EVAM-COVID na cidade de Manaus. Trata-se de um relato de caso exclusivo sobre a atuação da equipe de enfermagem no planejamento de embarque que, didaticamente, foi dividido em três etapas: preparo dos equipamentos e materiais aeromédicos, configuração da aeronave e configuração dos leitos.

\section{Preparo dos equipamentos e materiais aeromédicos}

Na fase pré-transporte, prever e prover os materiais e equipamentos a serem utilizados na missão, pois a configuração interna é bastante variável, dependendo do tipo da aeronave. Médico e enfermeiro devem realizar em conjunto a organização dos equipamentos, materiais e medicamentos, estabelecendo sua disposição na aeronave (Scuissiato et al., 2012).

Portanto, essa etapa do planejamento de embarque é fundamental para a segurança do paciente, uma vez que garante que os recursos destinados para os cuidados em voo estarão disponíveis caso sejam necessários. Neste sentido foi realizado o teste sistemático de todos os equipamentos aeromédicos (bombas infusaras, respiradores mecânicos, aspiradores portáteis, monitores multiparametros, entre outros), garante-se que todos os equipamentos em voo estão em pleno funcionamento (Agência Nacional de Vigilância Sanitária, 2021).

Além dessa atividade a equipe, ainda em solo, procedeu a checagem da quantidade e a validade de todos os insumos e medicamentos constantes na mochila de medicação. No transporte de pacientes em estado crítico, é importantíssimo ter quantidade suficiente de medicamentos com ação vasoativa e sedativa para a preparação de drippings durante o voo.

\section{Configuração da aeronave}

Com antecedência significativa, iniciou-se o trabalho da equipe de enfermagem para o preparo da configuração da aeronave. O que orienta essa configuração é a quantidade, as condições clínicas e a gravidade dos pacientes que estarão no transporte aeromédico (Luz, Seixas \& Silva, 2018).

Uma preocupação no transporte de passageiros potencialmente contaminados, principalmente quando a via de contaminação é aérea, é a posição dos mesmos em relação ao fluxo de ar da aeronave. Nesses casos, é importante separar a aeronave em zonas de contaminação (Borges et al. 2020).

A zona quente é o local destinado ao transporte do paciente, com todos os profissionais devidamente paramentados. A zona Morna é o local destinado para armazenar equipamentos e materiais para o uso no transporte aeromédico. Já a zona fria, abriga os pilotos, mecânicos e demais especialistas que necessitem estar no voo, considerada livre de contaminação (Ministério da Defesa, 2021).

Para missões de transporte aeromédico de paciente com COVID-19 em Manaus, a aeronave C-105 foi configurada com cockpit (cabine de pilotagem) como zona fria (destacada em verde), visto que o mesmo é isolado do restante da aeronave 
por uma porta que permanece fechada durante todo o percurso. A estação 1 da aeronave (destacada em amarelo) foi considerada como zona morna e nela permaneciam a tripulação do voo, a parte dos materiais aeromédicos e os acompanhantes dos pacientes que estavam sendo transferidos para outros estados. Já a zona quente ocupou as estações 2,3 e 4 (destacada em vermelho) e estava configurada com 04 leitos de UTI aéreo. Entre as zonas morna e quente da aeronave utilizou-se lonas para delimitá-las.

\section{Configuração dos leitos}

O transporte de pacientes deve ocorrer quando os benefícios esperados para ele excedem os riscos inerentes ao transporte e, também, quando o paciente necessita de cuidados que não existam no hospital onde está (Libardi, 2019).

Portanto, qualquer eventualidade que possa contribuir para a piora do caso clínico do paciente precisa ser abordada de forma qualificada pela equipe de enfermagem durante o voo.

A preparação do leito para o paciente em estado crítico aconteceu de forma individualizada. Quanto maior a gravidade do mesmo, maior será a quantidade de problemas que poderão advir de sua patologia. Foi realizado contato telefônico prévio com a equipe de enfermagem do hospital de destino para possibilitar a coleta de informações atualizadas sobre o estado geral do paciente e, consequentemente a antecipação de suas necessidades em voo.

Para facilitar a coleta de informações, nas missões realizadas pela Célula EVAM-COVID na cidade de Manaus, foi elaborado um texto padrão com informações importantes sobre o preparo dos pacientes para o voo e, ao mesmo tempo, colhendo informações pertinentes a configuração do leito. O referido texto, enviado por mensagem através do aplicativo WhatsApp, contemplava informações como: doenças pré-existentes, sinais vitais e parâmetros da ventilação mecânica e a concentração e velocidade das medicações infundidas em bombas infusaras. Após a coleta dessas informações, procedeu-se o preparo do leito com todos os pré-ajustes necessários, minimizando o estresse inicial de adaptação do paciente aos equipamentos.

Portanto segundo Borges et al., (2022) considera-se que conhecimentos, associados aos preceitos de segurança em saúde são importantes para o planejamento da assistência de enfermagem ao paciente que será transportado por aeronave, contribuindo assim para a continuidade do tratamento e reduzindo aos mínimos aceitáveis os possíveis danos advindos da atividade aérea.

\section{Conclusão}

O estudo traz uma abordagem dos cuidados específicos no pré-voo da evacuação aeromédica. Neste sentido, o enfermeiro desenvolve as ações de planejamento de embarque, com base na experiência da assistência a pacientes críticos e, participa da equipe multiprofissional durante o voo. Portanto, são fundamentais as habilidades de comunicação, tomada de decisão para manter a equipe unida em cooperação para o objetivo comum.

A experiência vivenciada pela enfermagem no planejamento dos transportes aeromédicos realizados na cidade de Manaus, gerou importante aprendizagem e segurança para a equipe. Espera-se que o relato desse caso seja relevante para a enfermagem aeroespacial compartilhando as estratégias de planejamento de embarque.

Os resultados deste estudo contribuem para reflexões para os Enfermeiros acerca da atuação da enfermagem na Evacuação Aeromédica, que necessita de recursos humanos devidamente treinados e preparados para este evento. Portanto, tratou-se de uma experiência que proporcionou aprendizado para a equipe e reflexões para mudanças de atitudes considerando os benefícios que proporciona ao paciente e, segurança para equipe desenvolver assistência de qualidade e de segurança. 
Research, Society and Development, v. 11, n. 3, e11311326437, 2022

(CC BY 4.0) | ISSN 2525-3409 | DOI: http://dx.doi.org/10.33448/rsd-v11i3.26437

Evidencia-se que produções de estudos que abordem transporte aeromédicos pela visão da enfermagem ocorre mais nos últimos anos e ainda há poucas produções acerca do tema. Com isso, sugerem-se que estudos voltados para a atuação do enfermeiro de bordo e como podem contribuir com a melhoria da assistência prestada ao paciente aerorremovido.

\section{Referências}

Agência Nacional de Vigilância Sanitária. (Brasil). (2021). Guia para Serviços de Transporte Aeromédico de Passageiros com COVID-19. Brasília http://antigo.anvisa.gov.br/documents/10181/6339783/Guia+n\%C2\%BA+53_2021+-+vers\%C3\%A3o+1\%2C+de+08+10+21.pdf/bb8b4be6-2b9e-4b0f-b02fcf47e8e5d9c5

Andreoli, R. (2021). O desenvolvimento do transporte aeromédico no Brasil diante da pandemia de Covid-19. Brasília. https://portal.airconnected.com.br/2021/10/06/o-desenvolvimento-do-transporte-aeromedico-no-brasil-diante-da-pandemia-de-covid-19/

Luz, A. M., Seixas, P. R., \& Silva, S. A. (2018). Atuação do enfermeiro no transporte aeromédico. PECIBES. 4(2). https://periodicos.ufms.br/index.php/pecibes/article/view/6928

Barreto, I. C. H. C., Costa, V., Ramos, R. F., Oliveira, L. G., Martins, N. R. A. V., Cavalcante, F. V., Andrade, L. O. M., \& Santos, L. M. P. (2021). Colapso na Saúde em Manaus: o fardo de não aderir às medidas não farmacológicas de redução da transmissão da COVID-19. Scielo Preprints. 1. https://preprints.scielo.org/index.php/scielo/preprint/view/10.1590/0103-1104202113114

Beard, L., Laz, P.; \& Tindal, M. (2016). Efeitos fisiológicos na transferência de pacientes críticos. Anaesthesia Tutorial of the week, Tutotial. 6(30),1-8 https://tutoriaisdeanestesia.paginas.ufsc.br/files/2016/06/330-Efeitos-fisiol\%C3\%B3gicos-na-transferencia-de-pacientes-criticos.pdf

Borges, L. L., Guimarães, C. C. V., Aguiar, B. G. C., \& Felipe, L. A. F. (2020). Enfermagem Militar na "Operação Regresso ao Brasil”: evacuação aeromédica na pandemia do coronavírus. Rev. Bras. Enferm. 73(Suppl 2),e20200297. https://doi.org/10.1590/0034-7167-2020-0297

Borges, L. L., Guilherme, F. J. A., Lima, R. A., Aguiar, B. G. C., \& Haberland, D. F. (2022). Conhecimentos essenciais de fisiologia aeroespacial necessários para atuação do enfermeiro no transporte aeromédico: revisão de literatura. Research, Society and Development. 11(2), e20911225713. http://dx.doi.org/10.33448/rsd-v11i2.25713

Faria, L. (2021). Força Aérea transporta mais de 500 pacientes da região Norte para outros estados Ministério da Defesa. Força Aérea Brasileira. Brasília. https://www.fab.mil.br/noticias/mostra/36920/

Força Aérea Brasileira. (Brasil). (2011). Avião da FAB transporta paciente classificado como suspeito de infecção por ebola. Brasília. https://www.fab.mil.br/noticias/mostra/23637/SA\%C3\%9ADE\%20-

\%20Avi \%C3\%A3o\%20da\%20FAB\%20transporta\%20paciente\%20classificado\%20como\%20suspeito\%20de\%20infec\%C3\%A7\%C3\%A3o\%20por\%20ebola

Força Aérea Brasileira. (Brasil). (2021). Aeronaves da FAB realizam mais de 1600 horas de voo em apoio à Região Norte. Brasília. https://www.fab.mil.br/noticias/mostra/36961/\#: :text=O\%20envolvimento\%20das\%20aeronaves\%20KC,)\%2C\%201.600\%20horas\%20de\%20voo

Kerr, L. R. F. S., Kendall, C., Almeida, R. L. F., Ichihara, M. Y., Aquino, E. M. L., Silva, A. A. M., Ximenes, R. A. A., Albuquerque, M. F. P. M., AlmeidaFilho, N., Souza, R. F., Filho, S. P. B., Souza, W. V., \& Barreto, M. L. (2021). COVID-19 in northeast Brazil: first year of the pandemic and uncertainties to come. Revista de Saúde Pública [online]. 55, 35. https://doi.org/10.11606/s1518-8787.2021055003728

Lana, R. M., Coelho, F. C., Gomes, M. F. C., Cruz, O. G., Bastos, L. S., Villela, D. A. M., \& Codeço, C. T. (2020) Emergência do novo coronavírus (SARSCoV-2) e o papel de uma vigilância nacional em saúde oportuna e efetiva. Cadernos de Saúde Pública. 36(3), e0019620. https://doi.org/10.1590/0102$311 \mathrm{X} 00019620$

Libardi, M. B. O. (2019). Transporte do Paciente Crítico e Resgate Aeromédico. Brasília. Especialização [Pós-Graduação Lato Sensu em Enfermagem Aeroespacial, Universidade Unyleya]. https://www.passeidireto.com/arquivo/49786392/transporte-do-paciente-critico-e-resgate-aeromedico

Ministério da Defesa. (Brasil). (2021). Nota técnica $N^{o}$ 015, de 10 de fevereiro de 2021. Orientações relativas ao transporte aeromédico de pacientes com covid-19 no âmbito do Exército Brasileiro. Brasília. http://www.dsau.eb.mil.br/index.php/coronavirus-diretrizes-e-notas-tecnicas/category/568-notas-tecnicascovid-19?download=1130:nota-tecnica-n-015

Ministério da Saúde (Brasil). (2020). Portaria $n^{\circ} 454$, de 20 de março de 2020. Declara, em todo o território nacional, o estado de transmissão comunitária do coronavírus (Covid-19). https://www.in.gov.br/en/web/dou/-/portaria-n-454-de-20-de-marco-de-2020-249091587

Orellana, J. D. Y., Cunha, G. M., Marrero, L., Horta, B. L., \& Leite, I. C. (2020). Explosão da mortalidade no epicentro amazônico da epidemia de COVID-19. Cad. Saúde Pública. 36(7), e00120020. http://dx.doi.org/10.1590/0102-311X00120020

Passos, I. P. B. D., Toledo, V. P., \& Duran, E. C. M. (2011). Transporte aéreo de pacientes: análise do conhecimento científico. Rev. Bras. Enferm. 64(6), 1127-1131. https://doi.org/10.1590/S0034-71672011000600021

Pérez, C., García, G., Chacón, S., Marín, M., López, S., López, S., Miguel, C., \& Vañó, P. (2017). Aerotransporte de pacientes con alto riesgo de contaminación por enfermedad infecciosa, a propósito de tres casos. Sanid. Mil. 73(1), 46-56. https://scielo.isciii.es/pdf/sm/v73n1/informes2.pdf

Rocha, F. (2021). Operação COVID-19. Brasília. https://issuu.com/portalfab/docs/aerovisao_268_abr_mai_jun_2021 
Research, Society and Development, v. 11, n. 3, e11311326437, 2022

(CC BY 4.0) | ISSN 2525-3409 | DOI: http://dx.doi.org/10.33448/rsd-v11i3.26437

Scuissiato, D. R., Boffi, L. V., Rocha, R. R., Montezeli, J. H., Bordin, M. T., \& Peres, A. M. (2012). Compreensão de enfermeiros de bordo sobre seu papel na equipe multiprofissional de transporte aeromédico. Rev. Bras. Enferm. 65(4), 614-20. https://doi.org/10.1590/S0034-71672012000400010

Yoshida, W. B. (2007). Redação do relato de caso. J. vasc. bras. 6(2), 112-113. https://doi.org/10.1590/S1677-54492007000200004

Ventura, D. F. L., Perrone-Moisés, C., \& Martin-Chenut, K. (2021). Pandemia e crimes contra a humanidade: o "caráter desumano" da gestão da catástrofe sanitária no Brasil. Revista Direito e Práxis 12(3), 2206-2257. https://doi.org/10.1590/2179-8966/2021/61769 